\title{
Low levels of molecular agents as a cause of emotional disturbances in personality and the prophilactic use of molecular biocybernetic clavitherapy in the elimination of the need to harm others, suicidal tendencies, terrorism and geopolitical conflicts
}

\section{Perspective}

In the current state of interpersonal relationships there are far too many cases of the need to cause harm to others. It should be pointed out that the most common cause of this need to cause harm does not arise solely from the lack of suitable education in the field of coping with difficult social and existential conditions. In my analysis the psychological roots lie in shortages of molecular agents. A similar condition applies in the case of the rise in suicidal tendencies which to a large extent are also caused by shortages of molecular agents. The studies I have conducted over many decades in the area of the application of molecular cybernetic clavitherapy confirm in individual cases and to a high degree the successfulness and permanency of the rebuilding of resistance to psychological crises and thus elimination of the drive to harm others and also the elimination of the tendency to suicidal mania.

The above also applies in the case of terrorism which is a brutal weapon in a cold unemotional world divided by wealthy global corporations into a poor South and wealthy North. We need to bear in mind that the migration of the poor from underdeveloped countries to the wealthy North will escalate. And with these migrants potential terrorists will move. These potential terrorists will be ready to commit suicide in the name of other's causes and beliefs. The cause of this state also lies in historical factors when the majority of rich Northern countries used colonial policies and slavery to exploit poorly organised, helpless populations in the South. The North inspired revolutions in the Arab world at the turn of the 20th and 21st Century. At the time I tried to interest various organisations and social bodies in the rich North in my book Eden in the 21st Century. In this book I describe the resolution of old hurts and justified prejudices of the poor societies of the South as relatively insignificant and the fact that they are owed financial compensation for the denigration of their societies and the theft of natural resources.

I am conscious of the need to conduct systematical studies of the process of regeneration of deficits of molecular agents which I have identified and described in algorithms of the network of biocybernetic activity as these relate to particular personality, characterological and emotional disorders for the purposes of statistical analysis of various age groups. A significant example is the geopolitical positioning of Poland which caused numerous wars and even the fact that Poland ceased to exist for 123 years partitioned amongst its then three neighbours. I am convinced that the following description of the amelioration of deficiencies of molecular agents, coupled with
Volume 4 Issue 6 - 2016

\author{
Ferdynand Barbasiewicz \\ Ferdynand Barbasiewicz, The creator of the method, Poland
}

Correspondence: Ferdynand Barbasiewicz, The creator of the method, Poland, Tel +48227298053,

Email centrum@klawiterapia.com

Received: September 30, 2016 | Published: December 30, 2016

education and psychotherapy is the right vector in bearing help in the enumerated disorders leading to better conflict and suicide free balanced social coexistence in the future. The proposal to conduct studies in these areas can be an important guarantor of proper personal development free of aggression and suicide.

If you consider that the pragmatic introduction of psychotherapeutical support linked with Clavitherapy as a realistic response to historically conditioned reality and lack of educational, existential and emotional support and development of children please do read on.

There are a multitude of negative personality, characterological and emotional defects including bipolar disorder which are conditioned psychologically and encoded across generations endogenetically in the DNA at the core of cells at all endogenetic levels of mankind (also in domesticated animals) as a result of evolution over manymillions of years. Educationalists and clinical psychology try to deal with this genetic conditioning through extensive psychotherapy and psychiatric treatment. These therapeutic actions are contrary to logic. The escalating problem of lack of preparation and irrational failures in dealing with the resolution of conflict whose consequence is the need to cause harm when faced with difficult existential environments and in the case of those with significantly reduced ability to mitigate stress and frustration lead to depression and even suicide. In the light of this simplified analysis of the problem we can extrapolate a failure by traditional therapy to understand fully interpersonal behaviour. And in the case of the pragmatic resolution of geopolitical conflict which in the hands of incompetent politicians usually end in military action which destroy people and their environment. Does this have to be at the conscious interpersonal condition of mankind in the 21 st Century? Please see the enclosed appeal to world leader who will participate in this Autumns United Nations session. 
Given that the wisdom of NATURE has endowed the human body with hundreds of thousands of biologically active points, whose activation successfully eliminates personality, emotional and health disorders an aware PERSON should with humility take advantage of this universal gift of NATURE.

A clinical proof confirming the effect of shortfalls of molecular agents in causing emotional instability can be seen in the use of lithium in the reduction of symptoms in certain types of bipolar affective disorders. Until such time that molecular and even sub molecular science completes the quantification and qualification of the diagnostic significance of the 3 million odd different endogenetic molecular agents and the the some 10million neurochemical transmitters as well as specialist transmitters (in this micro cosmos there are also some 100,000 selective antibodies which without the intervention of medicines allow for the eradication of infections) we cannot question the effectiveness of the algoriths of the biologically active pressure points in the endogenetic removal of more than 1,100 types of personality disorders and illnesses set out in my book on clavitherapy and the atlas of clavitherapy. And if we add to these discrete processes regulating psychogenetically disordered endogenetic homeostasis of the human body the evolutionary conditioning permanently encoded in the DNA over millions of years we have the possibility of biocybernetically restoring nerve transmissions within the brain and nerve connections damaged by demyelinisation. We can also treat early stage malignant cancers at the level of the dermis and connecting tissue resulting in melanoma spots, moles and disfigurements and treat the straight line spread of cancerous cells and markers which block blood flow and in early stages cause itching leading on to localised pain and damage to nerves. These changes lead to paralisis of the nerves causing the blocking of physical functionality and loss of movement at all endogenetic levels as disclosed by clavitherapy. We thus have a fantastic ability, using molecular biocybernetic clavitherapy, to liquidate psychological, emotional, psychosomatic, infectious and cancerous disorders including malignant tumours and metastatis. In the current state of interpersonal relationships there are far too many cases of the need to cause harm to others. It should be pointed out that the most common cause of this need to cause harm does not arise solely from the lack of suitable education in the field of coping with difficult social and existential conditions. In my analysis the psychological roots lie in shortages of molecular agents. A similar condition applies in the case of the rise in suicidal tendencies which to a large extent are also caused by shortages of molecular agents. The studies I have conducted over many decades in the area of the application of molecular cybernetic clavitherapy confirm in individual cases and to a high degree the successfulness and permanency of the rebuilding of resistance to psychological crises and thus elimination of the drive to harm others and also the elimination of the tendency to suicidal mania.

The above also applies in the case of terrorism which is a brutal weapon in a cold unemotional world divided by wealthy global corporations into a poor South and wealthy North. We need to bear in mind that the migration of the poor from underdeveloped countries to the wealthy North will escalate. And with these migrants potential terrorists will move. These potential terrorists will be ready to commit suicide in the name of other's causes and beliefs. The cause of this state also lies in historical factors when the majority of rich Northern countries used colonial policies and slavery to exploit poorly organised, helpless populations in the South. The North inspired revolutions in the Arab world at the turn of the 20th and 21st Century. At the time I tried to interest various organisations and social bodies in the rich North in my book Eden in the 21st Century. In this book I describe the resolution of old hurts and justified prejudices of the poor societies of the South as relatively insignificant and the fact that they are owed financial compensation for the denigration of their societies and the theft of natural resources.

I am conscious of the need to conduct systematically studies of the process of regeneration of deficits of molecular agents which I have identified and described in algorithms of the network of biocybernetic activity as these relate to particular personality, characterological and emotional disorders for the purposes of statistical analysis of various age groups. A significant example is the geopolitical positioning of Poland which caused numerous wars and even the fact that Poland ceased to exist for 123 years partitioned amongst its then three neighbours. I am convinced that the following description of the amelioration of deficiencies of molecular agents, coupled with education and psychotherapy is the right vector in bearing help in the enumerated disorders leading to better conflict and suicide free balanced social coexistence in the future. The proposal to conduct studies in these areas can be an important guarantor of proper personal development free of aggression and suicide.

If you consider that the pragmatic introduction of psychotherapeutical support linked with Clavitherapy as a realistic response to historically conditioned reality and lack of educational, existential and emotional support and development of children please do read on.

There are a multitude of negative personality, characterological and emotional defects including bipolar disorder which are conditioned psychologically and encoded across generations endogenetically in the DNA at the core of cells at all endogenetic levels of mankind (also in domesticated animals) as a result of evolution over many millions of years. Educationalists and clinical psychology try to deal with this genetic conditioning through extensive psychotherapy and psychiatric treatment. These therapeutic actions are contrary to logic. The escalating problem of lack of preparation and irrational failures in dealing with the resolution of conflict whose consequence is the need to cause harm when faced with difficult existential environments and in the case of those with significantly reduced ability to mitigate stress and frustration lead to depression and even suicide. In the light of this simplified analysis of the problem we can extrapolate a failure by traditional therapy to understand fully interpersonal behaviour. And in the case of the pragmatic resolution of geopolitical conflict which in the hands of incompetent politicians usually end in military action which destroy people and their environment. Does this have to be at the conscious interpersonal condition of mankind in the 21 st Century. Please see the enclosed appeal to world leader who will participate in this Autumns United Nations session.

Given that the wisdom of NATURE has endowed the human body with hundreds of thousands of biologically active points, whose activation successfully eliminates personality, emotional and health disorders an aware PERSON should with humility take advantage of this universal gift of NATURE.

A clinical proof confirming the effect of shortfalls of molecular agents in causing emotional instability can be seen in the use of lithium in the reduction of symptoms in certain types of bipolar affective disorders. Until such time that molecular and even sub molecular science completes the quantification and qualification of the diagnostic significance of the 3 million odd different endogenetic molecular agents and the some 10 million neurochemical transmitters 
as well as specialist transmitters (in this micro cosmos there are also some 100,000 selective antibodies which without the intervention of medicines allow for the eradication of infections) we cannot question the effectiveness of the algorithms of the biologically active pressure points in the endogenetic removal of more than 1,100 types of personality disorders and illnesses set out in my book on clavitherapy and the atlas of Clavitherapy. And if we add to these discrete processes regulating psychogenetically disordered endogenetic homeostasis of the human body the evolutionary conditioning permanently encoded in the DNA over millions of years we have the possibility of biocybernetically restoring nerve transmissions within the brain and nerve connections damaged by demyelinisation. We can also treat early stage malignant cancers at the level of the dermis and connecting tissue resulting in melanoma spots, moles and disfigurements and treat the straight line spread of cancerous cells and markers which block blood flow and in early stages cause itching leading on to localised pain and damage to nerves. These changes lead to paralisis of the nerves causing the blocking of physical functionality and loss of movement at all endogenetic levels as disclosed by Clavitherapy. We thus have a fantastic ability, using molecular biocybernetic Clavitherapy, to liquidate psychological, emotional, psychosomatic, infectious and cancerous disorders including malignant tumours and metastatis. Please refer to the simple method to destress individuals suffering from personality, character and emotional disorders using resocialisation techniques as well as neurophysiologic procedures referencing the enclosed algorithms which map the network of biocybernetic activity. Simple targeted non invasive stimulation which can be performed using simple wooden skewers or toothpicks of selected biologically active receptors on the skin surface, collagen fibres containing nerve endings, and the nervous system itself. The disorders are cancelled by regulating acquired shortfalls of endogenetic molecular agents with the simultaneous rebuilding of resistance at the psychological and immunological sub cellular level. The alpha numeric referencing relates to the algorithms describing the networks of the body.

On the basis of discussions with the renowned criminologist and founder and long time chairman of the Polish Society of Psychological Hygiene, and current chairman of the Polish Suicide Foundation, Professor Brunon Hołys and thanks to the mentoring of Profesor Antoni Florkowski, a psychiatrist at the Collegium Medicum of the Jagiellonian University of Kraków Doctor of Psychiatry Małgorzata Jabłońska has undertaken a doctoral thesis "Evaluation of psychiatric and criminal endogenetic factors in pathological behaviour. The thesis will be based on empirical studies of the use of clavitherapy alogorithms in the treatment of molecular agent deficiencies in the age group 10-13 of children with a pathological need to cause harm.

How does the theory of clavitherapy explain the existence of molecular and submolecular defincies as a cause of personality and emotional disorders including certain types of bipolar disfunction?

Children, young adults and grownups who do not have suitable educational and psychological training when facing different types of stress, frustration, long term irritability arising from unresolved conflicts, emotional affectation, psychological dissonance and depression suffer significant lowering of psychological resistance. The enumerated emotional conditions lead to destruction of endogenetic molecular agents whose deficit manifest itself in personality and character disorders which can lead to irrational bipolar behaviour. The described personality, character and emotional disorders if not treated amongst by prophylactic, psychotherapeutic and Clavitherapy methods lead to psychosomatic disorders, addiction and physical illnesses including infectious conditions resistant to medication and to reduced immunological resistance.

Below is a listing of selected algorithms of the network of biologically active points exploited in biocybernetic molecular Clavitherapy. The selected points should be stimulated as set out in my Clavitherapy manual and the atlas of pressure points.

Educationalists, clinical psychologists and psychiatrists classify the listed disorders as personality, character and emotional disorders which if left untreated can lead to criminal activity or attempted suicide. As a result of lack of knowledge and mapping of molecular agents the disorders are treated as the end product of inadequate education. The relevant stimulation points are amongst others:

Bad humour Pm-65, N: 7 and 8, PaM-3

Permanent dissatisfaction N-7, 3

Hate of people aften aggressive N-8, Wż-20, GRT 20, U-1

Need to harm others W: 5 and 9

Anger and feelings of helplessness O-8

Eccentric behaviour GRT-21

Character pathology GRT -12

Character pathology particularly in badly behaved, vicious, lazy and obstinate children ST-2, W; 5 and 9

Dictatorial tendency GRT-15

Anxiety S-7, Pm-62, O: 3, 5 and 6, Po-10, Wż 25 and 40, W: 3 and 8, GRT: 11 and 23. GRP-15 and NP-28

\section{General exhaustion GRT-14}

Permanent tiredness physical fatigue Jg; 4 and 11, Ż-36, ST-6, Po-4, Wż 20 and 39, W-3, GRT: 14 and 23, GRP-6

Psychological exhaustion O-8

Lack of concentration ST-2, GRT-20, PaM-1

Mental retardation ST-2

Memory dysfunction ST-2, GRT-20

Memory disorders PaM-1

Loss of memory O-9

Lowering of memory S-3, Pm: 15 and 43, O-9, GRT: 8, 11, 19 and 20

State of psychophysical stimulation S-7, Po-10, Wż: 8, 24 and 25, W: 3 and 8 , GRP-15

To achieve general control of psyche, calming PaM-3

Psychological imbalance N-27

Obsessions ST-5

Nightmares Ż-45, ST-1, Wż: 43 and 44, U-29

Abnormal fear GRT: 11, 14 and 24, GRP-14

Emotional instability GRT-24

Will emotional disorder Pm: 15 and 16

Sleepiness Jg: 13 and 14, Pm-12, GRT-22 
Inability to rest NP-28

Vegetative neurosis ST-2, GRP 11 and 12, U-121

Juvenile neurosis Jg-16

Fear neurosis Ż-36, Jg-4, ST:2 and 3, S-7, Jc:3 and 7, Pm:14,15,19 and $67, \mathrm{O}: 3,4,5,6$ and 9, Po-4, GRT-12,

GRP:14 and 15

Sexual neurosis U-58

Neurosis coupled with fear, sadness and longing P:3, 5 and 9, Jg-4 $\mathrm{S}: 5,7$ and 9, Jc-3, Pm-67, N:3, 24 and 25 and in the case of women O-9, GRT 4 and 26, GRP-4, U-87

Depressive neurosis P-9, Jg-4, Ż: 36,40 and 41, ST:1, 2, 3 and 4, S:3,5 and $9, \mathrm{Jc}: 3$ and 7, Pm:15 and 67, O:5, 6 and

7, Po:3, 4 and 5, Wż:34 and 40, W:8 and 13, GRT:12, 14, 15 and 23, GRP:4 ,5,6 and 14, U:55, 95 and 100

Reactive neurosis P-5, Ż-14, N-2, GRT-15, GRP: 14 and 15

Hysterical neurosis Jg-4, Ż-36, ST-6, S:1, 3, 4, 6 and 7 Pm:10 and 15, $\mathrm{N}: 1$ and 4, O:6 and 8, GRT:8 and 26, GRP-15, PaM:28,54,64, 86,115 and $118, \mathrm{U}: 51,55,87$ and 100

Menopausal neurosis U: 22, 23 and 58

Vegetative neurosis Jg-4, Ż-36, ST-2 and 6, S-7, Pm-15, O-6, Po-5, Po-5, GRT: 12 and 23 GRP: 5,11,12 and 15, U:34, 51, 55, 87,100 and 121

Neurostania Jg:4 and 11, Ż:36 and 45, ST-6, Jc: 7 and 14, Pm: 10, 14 , $15,18,22,23,31,32,33$ and 34, N:4, 6 and 9, O:4 and 7, Wż: 5, 6, 20, 23 and 39, GRT: 8, 11, 12,13, 17, 18 and 20, GRP:6, 12 and 14, PaM-85, U:29,33,36,87 and 100

Calming S-7, Pm-10, O: 3 and 6, Wż: 40, W: 2 and 3, GRT:11, 20 and 23, GRP-12, U:33, 34,55 and 87 Hypersensitivity S-7,

Disturbed sleep Pm-18,Wż-12, GRT:4 and24,GRP-12,U:33,87 and 100 Hypochondric neurosis P-9, Jg-4, Ż-36, ST-6, S-7, Pm-67, N-3, O-6, PaM-3, U:55, $87 \quad 100$ and 121 Sleeplessness P:6, 9 and 11, Jg-4, Ż:27 and 36, ST:1, 6 and 9, S-7, Pm:10 and 62, N:3 and 6, O:6 and 7, Po:5 and 16. Wż-12, GRT: 4, 18 and 24, PaM:1 and 13, NP:27 and 28

General nervous illnesses Ż: 23 and 24, ST-1, Jc:5 and 7, Pm-60, O-8, Wż-39, GRT:12, 13, 14, 15 and 16, PaM:1, 28, 55, 121, 122, 125, 126, $143,149,166,167,168,169,170$ and 171

Illnesses of the nervous system P-5, U-34 (core of the brain)

Psychoneuroses GRT: 20, 23, and 26, GRP-24

Reactive psychoses Ż-45, S: 3 and 7, Pm-18, N-4, O: 4, 6, 7 and 8, Po:2 and 10, Wż-37, GRT:13, 16 and 17, GRP-15 Repetitive heart contractions of a nervous basis S: 5 and 9

Thirst of a nervous basis U-17

General feeling of cold ST-5, S-1, Pm-43, N-7

Illnesses of the sympathetic nervous system U-51

Blood flow to brain disorders Pm-10

Reduced blood flow to brain GRT-20, 21, 26, 27 and 28
Disturbances of blood flow in brain ST-2, U-130

Attack of giggles (manic laughter) O-7

Suicidal mania O-7

Halucinations Wż-24

Stroke aphasia S-9, O-8, NP: 19 and 78

Illnesses of the brain stem U-25

Illnesses of the thalamus and hypothalamus Po-20, U-26, A

Maniacal states Ż-40, Pm-65, GRT-1, GRP-14

Phobias Jg; 13 and 14, Pm-19, Po-19, GRT-12(nighttime)

Hysterical aphasia S-5, N-1

Forgetfullness NP-78

Amnesia S-9

State of fear GRP-14

Psychiatric disturbances GRT: 26, 27 and 28

Psychiatric illnesses ST: 1 and 2, Jc-7, GRT-15, PaM:1 and 55

Schizophrenia Ż: 4 and 40, U-121

The above enumerated disorders are on the result on the one hand of poisoning of the organism through negligence and on the other endogenetic burn out caused by metabolic fallout caused by stress, frustrations, irritation, affectations, psychological dissonances, depression, lack of oxygenation of the blood, lack of exercise and exposure to fresh air, inadequate nutrition, inability to realise self in work, monotony of daily life, caustic family relationships and poisoning of the organism. In general the enumerated causes lead to a shortfall and rarely a surplus of micro molecular endogenetetic agents which reflect significant changes in the psyche of the individual. Various stimulants and depressants such as tobacco and alcohol amongst others significantly increase negative effects. The above mentioned personality and emotional disturbances can be eliminated within a few days using biocybernetic stimulation. When using Clavitherapy it is suggested that the patient is under psychological care and counselling. The daily treatment lasts between 30 minutes and 3 hours over a period of 7 to 10 consecutive days dependent upon the type of disorder and will lead, without the use of drugs to removal of the disorder. Of course the individuals' material and existential environment must be improved and perspectives for a better life set out. Having in mind the ecological balance of the wisdom of nature, let us respect the gift of life and let us also share in the wellbeing and happiness of others - motto and mission of the author.

There are already more than 100 therapists with medical qualifications trained in the use of Clavitherapy. A number of masters' theses have been written on Clavitherapy and currently two doctoral theses are under way at medical universities. The first doctorate is the subject area set out above; the second concerns the treatment of posture using neurophysiological rehabilitation using Clavitherapy.

\section{Acknowledgements}

None.

\section{Conflict of interest}

The author declares no conflict of interest. 